\title{
ON THE DIVERGENCE OF FOURIER SERIES
}

\section{RICHARD P. GOSSELIN ${ }^{1}$}

1. By a well known theorem of Kolmogoroff there is a function whose Fourier series diverges almost everywhere. Actually, Kolmogoroff's proof was later generalized so that the Fourier series diverged everywhere $[2$, p. 175$]$; but we shall be concerned only with the almost everywhere theorem here. The proof involves rather severe restrictions on the orders of the partial sums which are shown to diverge. The following problem connected with this theorem was suggested to the author by Professor A. Zygmund. Given a sequence $\left\{p_{\nu}\right\}$ of positive integers increasing to $\infty$, can an integrable function $f$ on $(0,2 \pi)$ be constructed so that the partial sums of its Fourier series of order $p_{\nu}$ diverge almost everywhere ? The object of our paper is to give an affirmative answer to this question. Let $s_{p}(x ; f)$ denote the $p$ th partial sum of the Fourier series of the function $f$ at the point $x$.

THEOREM. Let $\left\{p_{\nu}\right\}$ be a sequence of integers increasing to $\infty$. Then there is an integrable function $f$ such that the sequence $s_{p_{\nu}}(x ; f)$ diverges almost everywhere.

2. Our construction of $f$ is a modification of that of Kolmogoroff. In the latter $f$ was made a sum of trigonometric polynomials of the following type:

$$
\frac{1}{n+1} \sum_{k=0}^{n} K_{m_{k}}\left(x-A_{k}\right), \quad A_{k}=\frac{4 \pi k}{2 n+1}
$$

where $K_{m}$ is the Fejer kernel of order $m$. The orders $m_{k}$ were made to satisfy the following conditions:
(a) $m_{0} \geqq n^{4}$;
(b) $m_{k+1}>2 m_{k}$;
(c) $2 n+1$ divides $2 m_{k}+1, k=0,1, \cdots, n$.

See $[1$, p. 70$]$.

Given $n$, from our sequence $\left\{p_{\nu}\right\}$, we choose $(2 n+1)^{2}$ distinct terms $p_{\nu(j)}, j=1,2, \cdots,(2 n+1)^{2}$ in their natural order so that (i) $p_{\nu(1)}$ $>2 n^{4}$, (ii) $p_{\nu(j)} \leqq(2 n)^{-2} p_{\nu(j+1)}, j=1,2, \cdots,(2 n+1)^{2}-1$. Dividing each number $2 p_{v(j)}+1$ by $2 n+1$ leaves a remainder $s_{j}$. Since there are only $2 n+1$ possible remainders, at least $n+1$ of the numbers

Presented to the Society November 30, 1957; received by the editors September 12' 1957.

1 This work was supported by the National Science Foundation through Research Grant NSF G-2789. 
must have the same remainder, say $s$. Let $2 p_{v(j)}+1=L_{j}+s$. If $s$ is even, the $L_{j}$ 's are odd. Let $2 M_{j}+1=L_{j}$ and $s=2 r$. Then $p_{\nu(j)}=M_{j}+r$. If $s$ is odd, the $L_{j}$ 's are even. We write $2 p_{v(j)}+1=\left(L_{j}-2 n-1\right)$ $+(s+2 n+1)$. Let $2 M_{j}+1=L_{j}-2 n-1$ and $2 r=s+2 n+1$. Then $p_{\nu(j)}=M_{j}+r$ with $2 M_{j}+1$ divisible by $2 n+1$. Designate the $p_{\nu(j)}$ 's by $q_{k}$ in their natural order, $k=0,1, \cdots, n: q_{k}=m_{k}+r$ with $2 m_{k}+1$ divisible by $2 n+1$ and $0 \leqq r<2 n+1$. Now

$$
m_{0} \geqq p_{\nu(1)}-r \geqq n^{4} ; \quad m_{k+1} \geqq 2 n^{2} m_{k}
$$

by (i) and (ii) respectively. Hence all the conditions (a), (b), and (c) above are met in constructing an acceptable function of type (1). We write

$$
\phi_{n}(x)=\frac{e^{i r x}}{n+1} \sum_{k=0}^{n} K_{m_{k}}\left(x-A_{k}\right)
$$

and investigate the $q_{j}$ partial sums of this trigonometric polynomial for $x$ in the interval $J_{j}=\left(A_{j}+n^{-1}(\log n)^{-1 / 5}, A_{j+1}-n^{-1}(\log n)^{-1 / 5}\right)$, $1 \leqq j<n-n^{1 / 2}$.

Writing the Fejer kernels in exponential form, we have

$$
\begin{aligned}
s_{m_{j}+r}\left(x ; \phi_{n}\right)= & \frac{e^{i r x}}{n+1} \sum_{k=0}^{j} K_{m_{k}}\left(x-A_{k}\right) \\
& +\frac{e^{i r x}}{2(n+1)} \sum_{k=j+1}^{n} \sum_{l=-m_{j}-2 r}^{m_{j}}\left(1-\frac{|l|}{m_{k}+1}\right) e^{i l\left(x-A_{k}\right)}
\end{aligned}
$$

it being understood that the term corresponding to $l=0$ is $1 / 2$. Let the first term on the right be denoted by $R_{1}(x)$. The second term on the right may be written as

$$
\begin{aligned}
R_{2}(x)+R_{3}(x)= & \frac{e^{i r x}}{2(n+1)} \sum_{k=j+1}^{n} \sum_{l=-m_{j}-2 r}^{-m_{j}-1}\left(1-\frac{|l|}{m_{k}+1}\right) e^{i l\left(x-A_{k}\right)} \\
& +\frac{e^{i r x}}{n+1} \sum_{k=j+1}^{n} s_{m_{j}}\left(x-A_{k} ; K_{m_{k}}\right) .
\end{aligned}
$$

By the method of $[1$, p. 71$]$ it follows that

$$
\begin{aligned}
R_{1}(x)+R_{3}(x)= & -e^{i r x} \sin \left[\left(m_{j}+1 / 2\right) x\right] \\
& \cdot\left\{\frac{1}{n+1} \sum_{k=j+1}^{n} \frac{m_{k}-m_{j}}{m_{k}+1} \frac{1}{2 \sin \left(A_{k}-x\right) / 2}\right\}+G(x)
\end{aligned}
$$

where $|G(x)|$ is bounded by a numerical constant independent of $n$ 
for $x$ in $J_{j}$. The factor in curly brackets of the first term on the right may be written

$$
\begin{aligned}
T(x)+U(x)= & \frac{1}{n+1} \sum_{k=j+1}^{n} \frac{1}{2 \sin \left(A_{k}-x\right) / 2} \\
& -\frac{1}{n+1} \sum_{k=j+1}^{n} \frac{m_{j}+1}{m_{k}+1} \frac{1}{2 \sin \left(A_{k}-x\right) / 2} .
\end{aligned}
$$

By condition (ii), $\left(m_{j}+1\right)\left(m_{k}+1\right)^{-1} \leqq n^{-2}$ for $k>j$ so that the sum on the right is bounded. Summarizing our results thus far, we have from (2) and (3)

(4) $s_{m_{j}+r}\left(x ; \phi_{n}\right)=R_{2}(x)-e^{i r x} \sin \left[\left(m_{j}+1 / 2\right) x\right] T(x)+G(x)+H(x)$

where $|G(x)+H(x)|$ is bounded by a constant independent of $n$.

In the expression defining $R_{2}(x)$ above, the terms involving $|l| / m_{k}+1$ lead to a function $I(x)$ which is less in absolute value than $4 r m_{j}\left(m_{j+1}+1\right)^{-1} \leqq 1$ by (ii). Hence, $R_{2}(x)-I(x)$ is

$$
\begin{aligned}
\frac{i e^{i r x}}{2(n+1)} \sum_{k=j+1}^{n} \frac{\exp \left[-i\left(m_{j}+1 / 2\right)\left(x-A_{k}\right)\right]}{2 \sin \left(A_{k}-x\right) / 2} \\
-\frac{i e^{i r x}}{2(n+1)} \sum_{k=j+1}^{n} \frac{\exp \left[-i\left(m_{j}+1 / 2+2 r\right)\left(x-A_{k}\right)\right]}{2 \sin \left(A_{k}-x\right) / 2} .
\end{aligned}
$$

Since $\left(m_{j}+1 / 2\right) A_{k} \equiv 0, \bmod 2 \pi$,

$$
\begin{aligned}
R_{2}(x)= & I(x)+\frac{i}{2} \exp \left[i\left(r-m_{j}-1 / 2\right) x\right] T(x) \\
& -\frac{i}{2} \exp \left[-i\left(r+m_{j}+1 / 2\right) x\right]\left\{\frac{1}{n+1} \sum_{k=j+1}^{n} \frac{e^{2 i r A_{k}}}{2 \sin \left(A_{k}-x\right) / 2}\right\} .
\end{aligned}
$$

Let the sum in curly brackets of the third term on the right side be denoted by $T_{1}(x)$. Clearly $\left|T_{1}(x)\right| \leqq T(x)$ for every $x$ in $J_{j}$ by (3). Combining with (4) gives

$$
s_{m_{j}+r}\left(x ; \phi_{n}\right)=i \exp \left[i\left(r+m_{j}+1 / 2\right) x\right] \frac{T(x)}{2}
$$

$$
\begin{aligned}
& -i \exp \left[-i\left(r+m_{j}+1 / 2\right) x\right] \frac{T_{1}(x)}{2} \\
& +G(x)+H(x)+I(x) .
\end{aligned}
$$

Let $x_{0}$ be a particular value of $x$ in $J_{j}$. From the definition of $J_{j}$ 


$$
\begin{aligned}
\left|T(x)-T\left(x_{0}\right)\right| & \leqq \frac{\pi^{2}}{4} \frac{\left|x-x_{0}\right| n^{2}(\log n)^{2 / 5}}{(n+1)} \\
& +\frac{\left|x-x_{0}\right|}{4(n+1)} \sum_{k=j+2}^{n} \frac{1}{\left[\sin \left(A_{k}-x\right) / 2\right]\left[\sin \left(A_{k}-x_{0}\right) / 2\right]} \leqq C(\log n)^{2 / 5} .
\end{aligned}
$$

For the same reasons, $\left|T_{1}(x)-T_{1}\left(x_{0}\right)\right| \leqq C(\log n)^{2 / 5}$. We set $T$ $=T\left(x_{0}\right), T_{1}=T_{1}\left(x_{0}\right)$, and $t_{j}=r+m_{j}+1 / 2$ and obtain from $(5)$

$$
s_{m_{j}+r}\left(x ; \phi_{n}\right)=\frac{i}{2} e^{i t_{j} x} T-\frac{i}{2} e^{-i t_{j} x} T_{1}+L(x)
$$

where $|L(x)| \leqq C_{1}(\log n)^{2 / 5}$. Now $T \geqq C_{2}(\log n)$ if $1 \leqq j<n-n^{1 / 2}$, $[1 ;$ p. 71$]$. If $\left|T_{1}\right| \leqq\left(1-(\log n)^{-1 / 2}\right) T$, then

$$
\left|s_{m_{j}+r}\left(x ; \phi_{n}\right)\right| \geqq C_{3}(\log n)^{1 / 2} \text {. }
$$

If not, put $T_{1}=e^{i \alpha}\left|T_{1}\right|$, where $\alpha$ depends on $j$ but not on $x$. The first two terms of the right side of (6) may be written

$$
\frac{i}{2} T\left\{e^{i t_{j} x}-e^{-i\left(t_{j} x-\alpha\right)}\right\}+i\left\{\frac{T-\left|T_{1}\right|}{2} e^{-i\left(t_{j} x-\alpha\right)}\right\} .
$$

Since $0 \leqq T-\left|T_{1}\right| \leqq(\log n)^{-1 / 2} T$, the second term in curly brackets does not exceed in absolute value $2^{-1}(\log n)^{-1 / 2} T$. The first term above is

$$
-T e^{i \alpha / 2} \sin \left(t_{j} x-\alpha / 2\right) .
$$

Thus, if $x$ belongs to $J_{j}, 1 \leqq j<n-n^{1 / 2}$, and if

$$
\left|\sin \left(t_{j} x-\alpha / 2\right)\right|>(\log n)^{-1 / 2}
$$

it follows from (6), (7), and (8) that

$$
\left|s_{m_{j}+r}\left(x ; \phi_{n}\right)\right| \geqq C_{4}(\log n)^{-1 / 2} T \geqq C_{5}(\log n)^{1 / 2} .
$$

Let $E$ be the set of $x$ which belongs to some $J_{j}$ for which (8) is satisfied. Here (9) holds. The acceptable $J_{j}$ have total measure not less than

$$
\left(n-n^{1 / 2}-2\right)\left(\frac{4 \pi}{2 n+1}-\frac{2}{n(\log n)^{1 / 6}}\right)
$$

which equals $2 \pi$ minus a term which is $O\left[(\log n)^{-1 / 5}\right]$. The part of them for which (8) is not satisfied is $O(\log n)^{-1 / 2}$.

3. The proof can be completed by familiar means. Choose a sequence $\left\{n_{\mu}\right\}$ going to $\infty$ sufficiently rapidly and write 


$$
f(x)=\sum_{\mu=1}^{\infty}\left(\log n_{\mu}\right)^{-1 / 4} \phi_{n_{\mu}}(x) .
$$

The details of what is meant by "sufficiently rapidly" are given in $[2$, p. 175]. From what has been shown in $\$ 2$, it follows that for almost every $x$ and for arbitrarily large $n, p_{\nu}$ can be found from our original sequence such that $\left|s_{p_{\nu}}(x ; f)\right| \geqq C_{6}(\log n)^{1 / 4}$. Appropriate modifications can be made in the construction so as to make $f$ real.

\section{REFERENCES}

1. G. H. Hardy and W. Rogosinski, Fourier series, Cambridge, 1956.

2. A. Zygmund, Trigonometrical series, Warsaw, 1935.

UNIVERSITY OF CONNECTICUT

\section{A PROBLEM OF RICHARD BELLMAN ${ }^{1}$}

\section{TOMLINSON FORT}

In the January 1957 number of the Bulletin of the American Mathematical Society, Richard Bellman proposes the following problem:

"Consider the Sturm-Liouville problem

$$
\begin{aligned}
u^{\prime \prime}+\lambda[f(x)+\epsilon g(x)] u & =0 \\
u(0)=u(1) & =0
\end{aligned}
$$

where $f(x)$ and $g(x)$ are continuous functions over $[0,1]$ with positive minima.

Let us regard $\lambda_{1}$, the smallest charactistic value as a function of $\epsilon$. Is it true that $\lambda_{1}$, is an analytic function of $\epsilon$ for $R(\epsilon) \geqq 0$ ? In general, where is the singularity nearest the origin $(\epsilon=0)$ ?"

In the present note the method of differences is used.

We shall consider $f(x)$ and $g(x)$ defined as continuous functions $-\eta \leqq x \leqq 1+\eta$ where $\eta>0$ is arbitrarily small. Any definition outside the original interval of definition will do. We shall assume $|f(x)|<N$, $|g(x)|<N$ where $N$ is constant.

Presented to the Society November 29, 1957; received by the editors September $3,1957$.

1 This research was supported by the United States Air Force through the Air Force Office of Scientific Research of the Air Research and Development Command under Contract AF 18(603-23). Reproduction in whole or in part is permitted for any purpose of the United States Government. 